\title{
Evidence That Vesicles Undergo Compound Fusion on the Synaptic Ribbon
}

\author{
Gary Matthews ${ }^{1}$ and Peter Sterling ${ }^{2}$ \\ ${ }^{1}$ Department of Neurobiology and Behavior, State University of New York, Stony Brook, Stony Brook, New York 11794-5230, and ${ }^{2}$ Department of \\ Neuroscience, University of Pennsylvania, Philadelphia, Pennsylvania 19104
}

\begin{abstract}
The ribbon synapse can release a stream of transmitter quanta at very high rates. Although the ribbon tethers numerous vesicles near the presynaptic membrane, most of the tethered vesicles are held at a considerable distance from the plasma membrane. Therefore, it remains unclear how their contents are released. We evoked prolonged bouts of exocytosis from a retinal bipolar cell, fixed within seconds, and then studied the ribbons by electron microscopy. Vesicle density on ribbons was reduced by $\sim 50 \%$ compared with cells where exocytosis was blocked with intracellular ATP- $\gamma \mathrm{S}$. Large, irregularly shaped vesicles appeared on the ribbon in cells fixed during repetitive stimulation of exocytosis, and in some cases the large vesicles could be traced in adjacent sections to cisternae open to the medium. The large cisternal structures were attached to the ribbon by filaments similar to those that tether synaptic vesicles to the ribbon, and they occupied the base of the ribbon near the plasma membrane, where normal synaptic vesicles are found in resting cells. We suggest that the cisternae attached to ribbons represent synaptic vesicles that fused by compound exocytosis during strong repetitive stimulation and, thus, that vesicles tethered to the ribbon can empty their contents by fusing to other vesicles docked at the presynaptic membrane. Such compound fusion could explain the extremely high release rates and the multivesicular release reported for auditory and visual ribbon synapses.
\end{abstract}

Key words: retina; ribbon synapse; retinal bipolar cell; synaptic transmission; exocytosis; neurotransmitter release

\section{Introduction}

Synaptic ribbons support high rates of continuous neurotransmitter release by tethering a large pool of releasable vesicles near the membrane at the active zone (Sterling and Matthews, 2005). But how does this arrangement facilitate release? The ribbon is generally thought to act as a conveyor belt that shuttles tethered vesicles toward fusion at the plasma membrane. Alternatively, the ribbon might simply hold vesicles near each other to promote compound fusion (where each vesicle fuses with an already fused vesicle, causing a wave of exocytosis to sweep up the ribbon) (Parsons and Sterling, 2003). This would deplete vesicles on the ribbon and leave infoldings of nearby plasma membrane, as has been observed near ribbons of tonically depolarized hair cells (Lenzi et al., 2002) and rods (Rao-Mirotznik et al., 1995). These infoldings could be interpreted as endocytic structures arising from bulk retrieval of individually fused vesicles; however, as Lenzi et al. (2002) pointed out, they could also represent vesicles coalesced by compound fusion. Such a mechanism might also explain the synchronous multivesicular release observed in

\footnotetext{
Received Aug. 14, 2007; revised April 3, 2008; accepted April 6, 2008.

This work was supported by National Institutes of Health Grants EY03821 (G.M.) and EY08124 (P.S.), and by the Office of the Vice President for Research at Stony Brook University. We thank Dr. Jian Li for sectioning and photographing the tissue, Wendy Akmentin for the images of manually tilted sections, and Dr. Christophe Paillart for carrying out some of the experiments in which release was stimulated by spontaneous calcium action potentials.

Correspondence should be addressed to Gary Matthews, Department of Neurobiology and Behavior, Life Sciences 550, State University of New York, Stony Brook, Stony Brook, NY 11794-5230. E-mail: gary.g.matthews@sunysb.edu.

DOI:10.1523/JNEUROSCI.0935-08.2008

Copyright $\odot 2008$ Society for Neuroscience $\quad$ 0270-6474/08/285403-09\$15.00/0
}

postsynaptic recordings at the hair cell and the rod bipolar synapse (Glowatzki and Fuchs, 2002; Singer et al., 2004).

We further investigated this problem in retinal bipolar cells by evoking long bouts of vesicle fusion, then fixing within seconds and studying the ribbons by electron microscopy. To control for vesicles released during fixation, we compared with cells with exocytosis blocked by dialysis with ATP- $\gamma \mathrm{S}$. Because the releasable vesicle pool estimated by electrophysiology matches the number of vesicles tethered to synaptic ribbons in retinal bipolar cells (von Gersdorff et al., 1996), depleting the pool should deplete vesicles from the ribbons, as has been observed at hair cell synapses (Lenzi et al., 2002). Indeed, Holt et al. (2004) observed depletion of vesicles docked at the plasma membrane after prolonged stimulation of retinal bipolar cells, but they found no change in the total number of tethered vesicles. Now, using different stimuli from Holt et al. (2004), we do find a significant reduction in the overall number of vesicles attached to ribbons in stimulated cells. Moreover, some of the attached vesicles were larger than in quiescent cells, and in terminals fixed during repetitive stimulation, large cisternae occupied the base of the ribbon and appeared to connect to both the ribbon and the extracellular space. The larger vesicles and large cisternae may arise from fusion of vesicles with other vesicles at ribbon synapses, which in turn suggests that the ribbon may achieve high release rates by facilitating such compound fusion.

\section{Materials and Methods}

Single large-terminal bipolar neurons were isolated from goldfish retina by mechanical trituration after enzymatic digestion (Heidelberger and 
Matthews, 1992). The external solution consisted of (in mM) $115 \mathrm{NaCl}$, $2.5 \mathrm{KCl}, 1.6 \mathrm{MgCl}_{2}, 2.5 \mathrm{CaCl}_{2}, 10$ glucose, and 10 HEPES, pH 7.4. For the measurement of $\left[\mathrm{Ca}^{2+}\right]_{\mathrm{i}}$, cells were incubated for 8-10 min in a solution containing $5 \mu \mathrm{M}$ fura- $2 \mathrm{AM}$ (Invitrogen), followed by $25 \mathrm{~min}$ in fura-free solution. $\left[\mathrm{Ca}^{2+}\right]_{\mathrm{i}}$ was calculated from the ratio of the emitted light at two excitation wavelengths as detailed by Heidelberger and Matthews (1992).

Activity was monitored by means of the changes in calcium in the synaptic terminal, initiated by spontaneous calcium action potentials. In some experiments, cells were instead stimulated by local superfusion with high-potassium solution ( $80 \mathrm{~mm} \mathrm{KCl}$, replacing $\mathrm{NaCl}$ ) applied via an application pipette placed under visual control near the terminal, and the application was terminated by removing the application pipette as soon as calcium was observed to increase (application duration $\sim 1 \mathrm{~s}$ ). In other experiments, single isolated terminals were maintained under voltage-clamp using a whole-terminal patch pipette and stimulated with 250 - $\mathrm{ms}$ voltage pulses from -60 to $0 \mathrm{mV}$ while monitoring membrane capacitance (Heidelberger et al., 2002).

For electron microscopy, a bipolar neuron or isolated bipolar cell synaptic terminal attached to a film of Aclar was recorded using fura-2 imaging or whole-cell patch clamp and then fixed by local superfusion with $2.5 \%$ paraformaldehyde plus $2.5 \%$ glutaraldehyde in $0.1 \mathrm{M}$ phosphate buffer from an application pipette with a tip diameter of $\sim 20 \mu \mathrm{m}$ that was placed within $15 \mu \mathrm{m}$ of the cell. After $10-30 \mathrm{~s}$, the bath fluid was replaced with fixative, a rectangular mark was etched into the Aclar film around the recorded cell using the superfusion pipette, and the dish was placed at $4^{\circ} \mathrm{C}$ overnight. Cells were then additionally fixed in $1 \% \mathrm{OsO}_{4}$ plus $1.5 \% \mathrm{~K}$ ferrocyanide in $0.1 \mathrm{~m}$ phosphate buffer, dehydrated, and embedded in Embed 812. The embedded sample was peeled from the Aclar film, glued to an Epon blank, and sectioned serially at $\sim 90 \mathrm{~nm}$. The rectangular mark etched into the Aclar after recording left a corresponding mark in the cured embedding medium to guide sectioning. Sequences of 5-10 sections were mounted on Formvar-coated slot grids, stained in methanolic uranyl and lead, and then photographed in an electron microscope $(80-120 \mathrm{kV})$ at $10,000-120,000 \times$. In some cases, photos were acquired with the specimen tilted $\pm 30^{\circ}$, in $10^{\circ}$ increments, to provide stereoscopic images. To construct stereo pairs, an image at the tilt angle that provided the best view of the object of interest was selected, and an image separated by $20^{\circ}$ was used for the other member of the pair. Photos or negatives were digitized and analyzed using NIH ImageJ or Adobe Photoshop 7.0 (Adobe Systems) software. Diameters of vesicles were measured using ImageJ by taking the length of a line drawn across the widest point of the vesicle, from midpoint to midpoint of the membrane profile. For analysis of large pleomorphic vesicles (see Fig. 7E), the surface area of ribbon-attached structures was determined using the area of a polygon drawn around the perimeter.

\section{Results}

\section{Stimulation reduced the number of vesicles attached}

\section{to ribbons}

To trigger exocytosis, a bipolar cell terminal was stimulated using spontaneously occurring calcium action potentials (Zenisek and Matthews, 1998), voltage-clamp depolarization under whole-cell patch clamp, or a puff of high external potassium applied through an application pipette placed near the cell. Figure $1 A$ shows a cell that fired a series of calcium action potentials, each of which produced a rise in intraterminal $\left[\mathrm{Ca}^{2+}\right]_{\mathrm{i}}$ measured using fluorescence of fura-2. The cell was then fixed a few seconds after the last calcium spike by placing an application pipette containing fixative near the terminal. For comparison, unstimulated bipolar cells like that illustrated in Figure $1 B$ were obtained by incubation with $5 \mu \mathrm{M}$ muscimol, which strongly activates $\mathrm{GABA}_{\mathrm{A}}$ chloride conductance and prevents calcium spikes (Paillart et al., 2003).

Examples of synaptic ribbons from stimulated and unstimulated bipolar cells are shown in Figure 2, $A$ and $B$, respectively. Qualitatively, visual inspection of the examples suggests that fewer synaptic vesicles were attached to ribbons of stimulated cells, an impression that was confirmed by counting the number

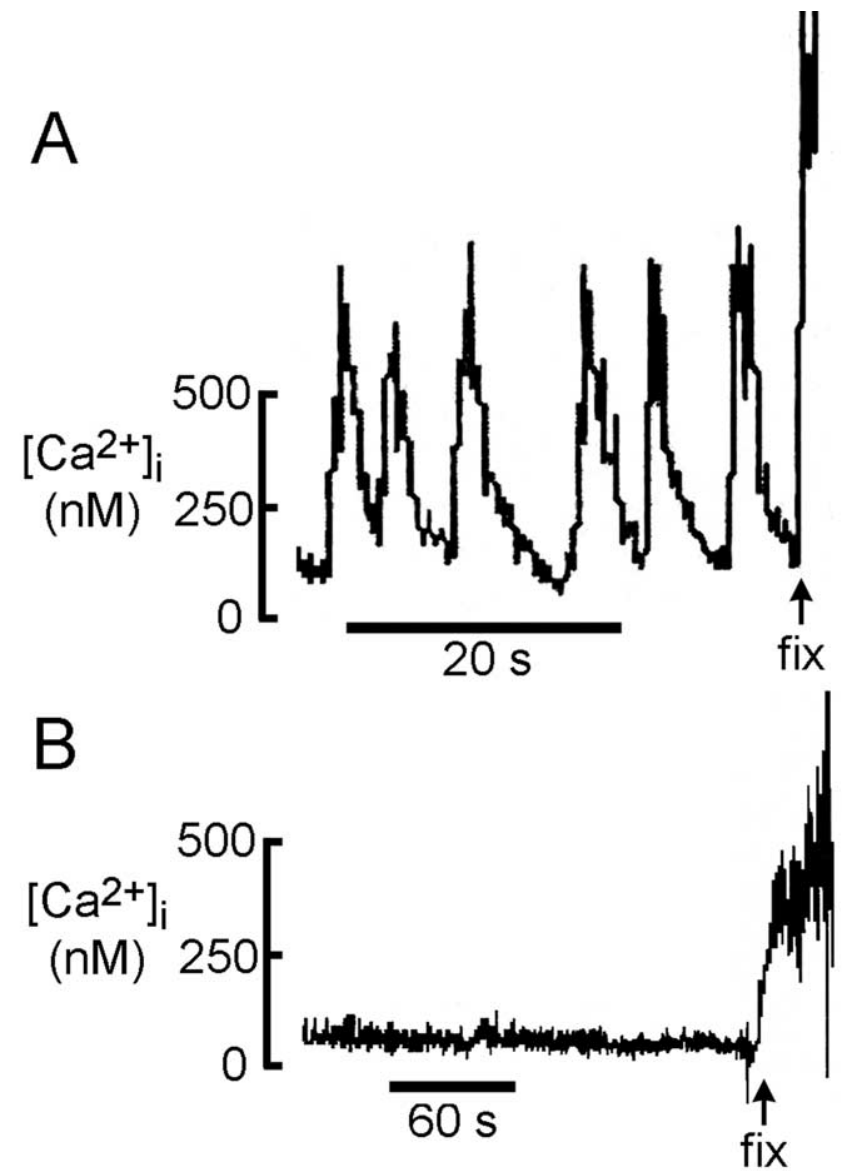

Figure 1. Isolated bipolar neuron fires spontaneous calcium action potentials, thereby raising the calcium concentration in the terminal. $\boldsymbol{A}$, Calcium spikes monitored with fura- 2 fluorescence. Fixative was applied at the indicated time via a pipette placed near the cell. $\boldsymbol{B}$, Calcium spikes were prevented by incubation in $5 \mu \mathrm{m}$ muscimol, but fixation raised calcium and probably caused some exocytosis.

of tethered vesicles in single thin sections at 70 ribbons in eight unstimulated terminals and at 38 ribbons in four stimulated terminals (Fig. 2D). In unstimulated cells, an average of $15.8 \pm 0.5$ vesicles (mean \pm SEM) were attached to ribbons, which is significantly greater than the $11.7 \pm 0.6$ attached vesicles/ribbon in stimulated cells $\left(p<10^{-6}\right)$. The depletion associated with stimulation was specific for vesicles tethered to ribbons, because the number of vesicles immediately surrounding the tethered group, but not attached, was similar in both conditions ("nontethered" vesicles) (Fig. 2D). This differs from the depletion of surrounding vesicles reported by Lenzi et al. (2002) at hair-cell synaptic bodies after prolonged depolarization; however, as discussed below, this vesicle population may already have been somewhat depleted even in the unstimulated cells.

\section{ATP- $\gamma \mathrm{S}$ blocked release associated with fixation}

We were concerned that calcium influx during fixation (Fig. $1 B$ ) might release neurotransmitter from the unstimulated cells, reducing the apparent difference between stimulated and unstimulated ribbons. For instance, aldehyde fixation evokes release of acetylcholine from motor nerve terminals, revealed by miniature end-plate potentials in the postsynaptic muscle cell (Smith and Reese, 1980). To minimize fixation-induced release, we prepared a set of cells that were fixed after dialysis with ATP- $\gamma \mathrm{S}$ through a whole-cell patch pipette, a treatment that inhibits vesicle priming 
A. Stimulated
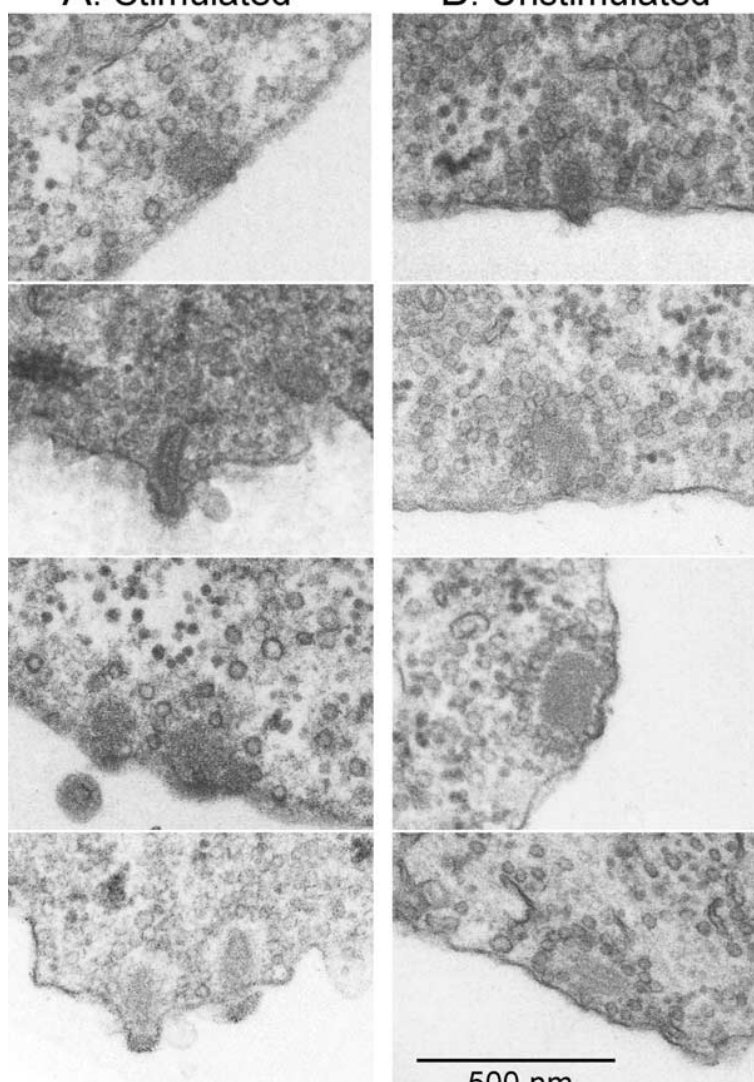

$500 \mathrm{~nm}$
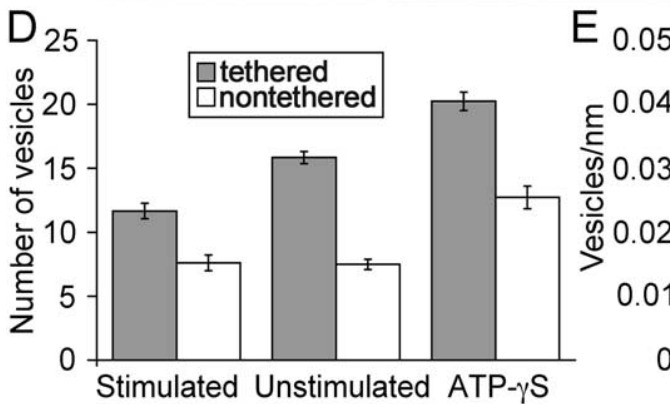
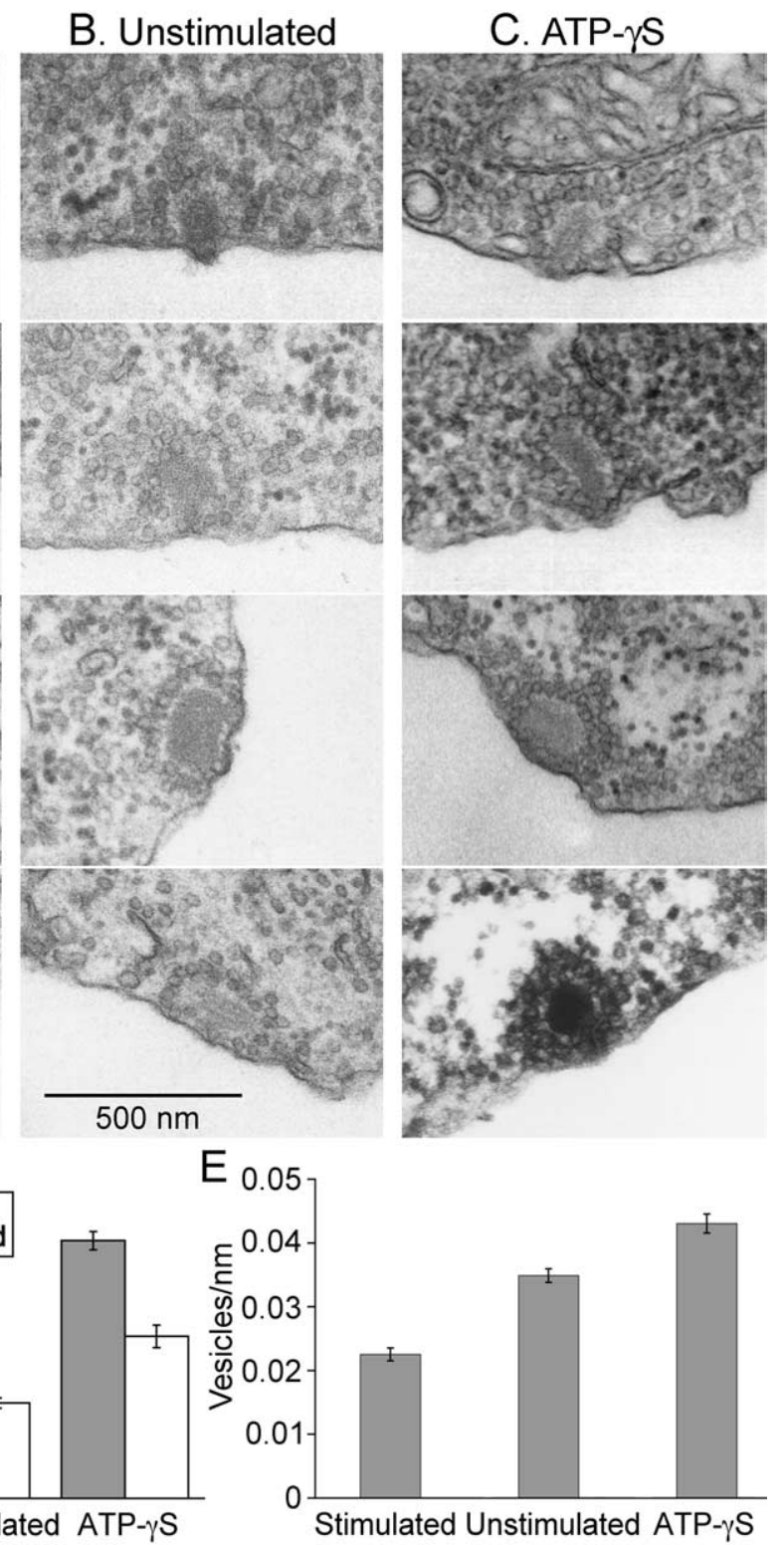

Figure 2. Calcium spiking reduced the number of synaptic vesicles on the ribbon. $A$, Stimulated cells were fixed within $5 \mathrm{~S}$ after a spike. $\boldsymbol{B}$, Calcium action potentials were blocked with muscimol, but fixation probably caused some exocytosis. $\boldsymbol{C}$, Exocytosis was blocked by substituting ATP- $\gamma S$ for ATP in the patch pipette in whole-cell recordings. The scale bar in $\boldsymbol{B}$ applies to all panels. $\boldsymbol{D}$, Average number of vesicles (in single thin sections) tethered to a ribbon (gray) and average number in the ring of nontethered vesicles (white) surrounding the tethered vesicles. $\boldsymbol{E}$, Two-dimensional density of vesicles attached to a ribbon in single thin sections (number of vesicles divided by ribbon perimeter; note that this measure is not expressed relative to maximum packing density) (Lenzi et al., 2002). Error bars show \pm 1 SEM.

and abolishes depolarization-triggered exocytosis in bipolar cell terminals (Heidelberger et al., 2002). Figure $2 C$ shows examples of ribbons and associated vesicles in these cells, which had significantly more vesicles tethered to ribbons. In cells treated with ATP $-\gamma \mathrm{S}$, the number of vesicles attached to ribbons in single thin sections averaged $20.4 \pm 0.7$ (mean \pm SEM; 37 ribbons in six cells), which was significantly greater $\left(p<10^{-5}\right)$ than in unstimulated terminals fixed without ATP- $\gamma \mathrm{S}$. As shown in Figure $2 D$, the number of nontethered vesicles surrounding the group attached to ribbons was also significantly higher $\left(p<10^{-5}\right)$ in cells dialyzed with ATP- $\gamma S$ compared with unstimulated and stimulated terminals. Therefore, inhibition of vesicle priming prevents depletion of both ribbon-tethered vesicles and the surrounding nontethered vesicles during aldehyde fixation.

\section{Stimulation decreased vesicle density on ribbons}

Synaptic ribbons vary somewhat in size and shape (Fig. 2), which influences the number of tethered vesicles they could accommodate. To control for this variation, we divided the number of attached vesicles by the measured perimeter of each ribbon to obtain vesicle densities for each condition (Fig. 2E). The pattern resembles that from raw vesicle counts: lowest density for stimulated cells, intermediate for unstimulated cells, and highest density for cells dialyzed with ATP- $\gamma$ S. All differences were highly statistically significant. Vesicles density on ribbons in stimulated cells was about half of that in cells dialyzed with ATP- $\gamma \mathrm{S}$. We take this as a lower bound for the stimulationinduced depletion of vesicles from the ribbon (see Discussion).

\section{Stimulation increased the number of large vesicles attached to ribbons}

In the course of these experiments, we noted that some vesicles tethered to ribbons were unusually large (Fig. 3A). Furthermore, the large vesicles seemed more prevalent in stimulated cells. To quantify this impression, we measured the diameter of all vesicles attached to ribbons in unstimulated cells, in cells fixed within $10 \mathrm{~s}$ after stimulation, and in cells dialyzed with ATP- $\gamma \mathrm{S}$. In the latter cells, the distribution of diameters for ribbon-tethered vesicles was fitted by a Gaussian function with a mean of $30.3 \mathrm{~nm}$ and SD of $3.7 \mathrm{~nm}$, similar to the previously reported average size of unattached synaptic vesicles in goldfish bipolar neurons (von Gersdorff et al., 1996). No unusually large vesicles were tethered to ribbons in ATP- $\gamma \mathrm{S}$ cells: only $0.26 \pm 0.16 \%$ (mean \pm SEM; $n=6$ ) of attached vesicles had diameters $>41 \mathrm{~nm}$, in keeping with the $0.2 \%$ expected for a Gaussian distribution with mean and SD of 30.3 and $3.7 \mathrm{~nm}$, respectively.

In stimulated cells and in unstimulated cells fixed without ATP- $\gamma \mathrm{S}$, the main peaks of the vesicle diameter distributions were no different from the ATP- $\gamma S$ cells, being fitted respectively by Gaussian functions with means of $30.7 \pm 4.4$ and $30.5 \pm 3.7 \mathrm{~nm}$ (Fig. $3 \mathrm{~B}, C$ ) (Gaussians were fitted to the histogram values on the negative side of the peak, and then reflected about the mean to generate the positive tail). Therefore, the size range of normal synaptic vesicles attached to ribbons is apparently the same in all three conditions.

However, unlike the finding with ATP- $\gamma S$ cells, more large vesicles were observed attached to ribbons in both stimulated and unstimulated cells than expected from the Gaussians fitted to the main peak of the histogram (Fig. $3 B, C$ ). Furthermore, stimulation significantly increased the proportion of these unusually large tethered vesicles. In eight stimulated cells, $5.6 \pm 0.8 \%$ of attached synaptic vesicles were $>41 \mathrm{~nm}$ in diameter, compared 

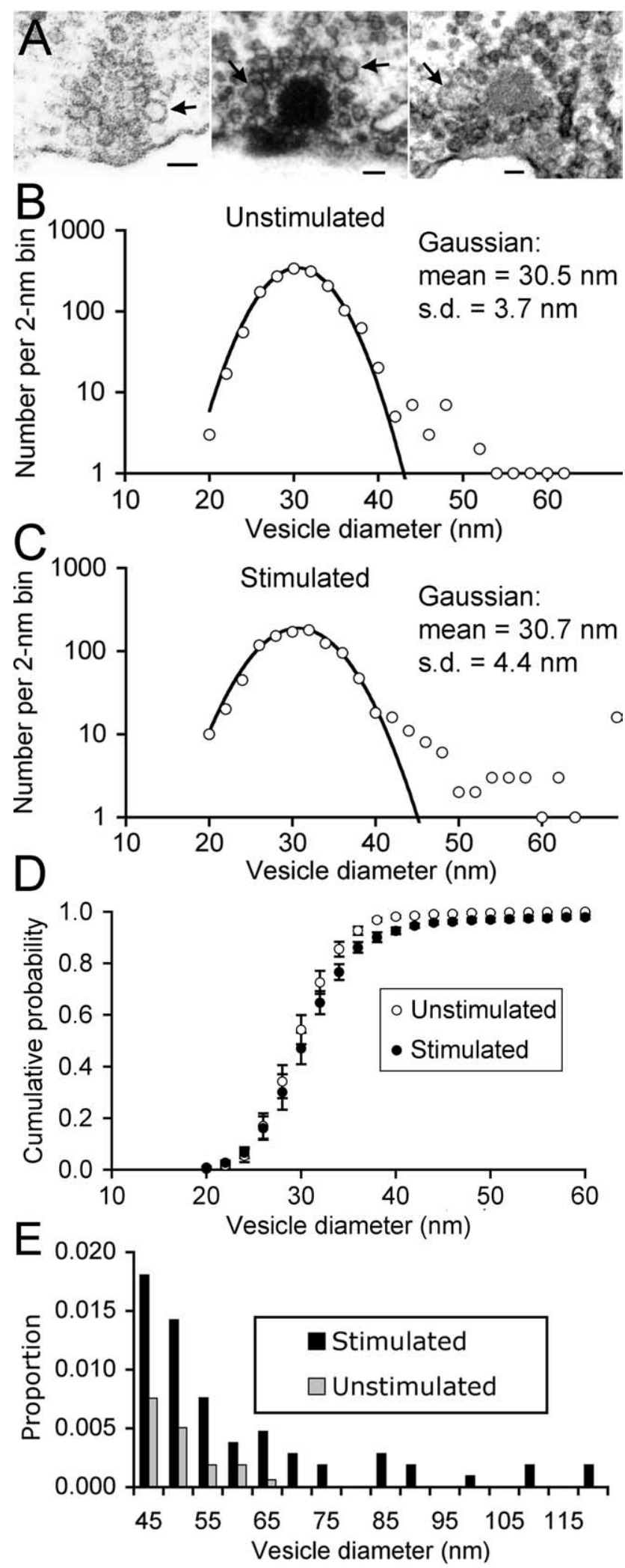

Figure 3. Stimulation increased the proportion of large vesicles attached to synaptic ribbons. $A$, Examples of large vesicles (arrows). Scale bars, $50 \mathrm{~nm}$. B, C, Size distributions of ribbonattached vesicles for unstimulated and stimulated cells. Solid lines are Gaussian functions fitted to the observed distribution to the left of the peak. $\boldsymbol{D}$, Cumulative distributions of ribbonattached vesicle diameter from eight stimulated cells (total of 1053 vesicles) and nine unstimulated cells (total of 1586 vesicles). Data points are averages across cells, and error bars show \pm 1 SEM. E, Proportion of total ribbon-attached vesicles accounted for by vesicles with diameters larger than $45 \mathrm{~nm}$ in stimulated cells (black) and unstimulated cells (gray). with $1.6 \pm 0.5 \%$ in nine unstimulated terminals $(p=0.001$ by two-tailed $t$ test). This 3.5 -fold increase in large, ribbon-attached vesicles after stimulation is reflected in the cumulative distributions of vesicle diameter for the stimulated and unstimulated cells (Fig. 3D). The difference between the distributions for stimulated and unstimulated cells is highly significant $\left(p<0.0001 ; \chi^{2}=\right.$ 20.05; $\mathrm{df}=2$; Kolmogorov-Smirnov two-sample test using $\chi^{2}$ approximation for large samples) (Siegel, 1956).

Figure $3 E$ shows the size distribution of the subset of vesicles $\geq 45 \mathrm{~nm}$ in diameter that were attached to synaptic ribbons in stimulated and unstimulated cells. This diameter was selected because no vesicles are projected in this size range from Gaussians fitted to the main peaks (Fig. $3 B, C$, solid lines). As expected from the fact that a single Gaussian function fits the distribution of vesicle diameters in the presence of ATP- $\gamma S$, no vesicles $\geq 45 \mathrm{~nm}$ in diameter were observed in these cells; therefore, no data are shown for the ATP- $\gamma S$ cells in Figure 3E. Ribbons in cells fixed soon after stimulation clearly had more of these large vesicles than ribbons in unstimulated cells, including some with diameters exceeding $100 \mathrm{~nm}$. Similar large vesicles have also been observed previously on photoreceptor ribbons [Schaeffer and Raviola (1978), their Fig. 7].

The large, ribbon-tethered vesicles that appear after stimulation might reflect vesicles that arose from endosomes during membrane retrieval, or they might reflect fusion of two or more normal-size vesicles with each other, i.e., compound fusion (Parsons and Sterling, 2003). The median size of the large subgroup of vesicles was $48 \mathrm{~nm}$, or $\sim 1.5$ times the diameter of normal vesicles. This median diameter is approximately consistent with fusion of two normal vesicles, which would be expected to produce an object with a diameter $\sqrt{ } 2$ larger than normal, if they fused completely to form a spherical vesicle.

By occupying more space on the ribbon than normal-size vesicles, the large vesicles that appear after stimulation would be expected to contribute to the stimulation-dependent decrease in vesicle density on ribbons discussed previously (Fig. 2). However, the large vesicles represent $\sim 6 \%$ of the total population of ribbon-associated vesicles, and their average size is such that they would occupy only slightly more space on ribbons than $30 \mathrm{~nm}$ vesicles. Therefore, the large vesicles could account for only a small part of the stimulation-induced depletion of vesicles at ribbons.

\section{After stimulation, some tethered vesicles connect to cisternae open to the medium}

In addition to large vesicles, we also observed frank infoldings of the plasma membrane associated with ribbons in terminals fixed within $5 \mathrm{~s}$ after spontaneous calcium spikes, exemplified in Figure 4. The successive sections in Figure $4 A$ show a ferritin-labeled invagination (Fig. 4A1) that in the next section (A2) connects to the ribbon via filaments similar to those that attach synaptic vesicles to the ribbon surface. Figure $4 B$ shows a large vesicle devoid of ferritin near the base of a ribbon, connected to the ferritinlabeled plasma membrane by a thin neck. A large, complex invagination associated with a ribbon is illustrated in Figure 4C. In three terminals fixed $<5 \mathrm{~s}$ after a calcium spike, $57 \%$ of ribbons (12 of 21) showed infoldings or invaginations of the plasma membrane at the base of the ribbon, but similar structures were observed at only $11 \%$ of ribbons ( 3 of 28 ) in unstimulated terminals $(p<0.001$, Fisher exact probability test). The presence of such invaginations and cisternae after stimulation has been reported previously in synapses of hair cells (Lenzi et al., 2002) and bipolar cells (Paillart et al., 2003; Holt et al., 2004). However, 


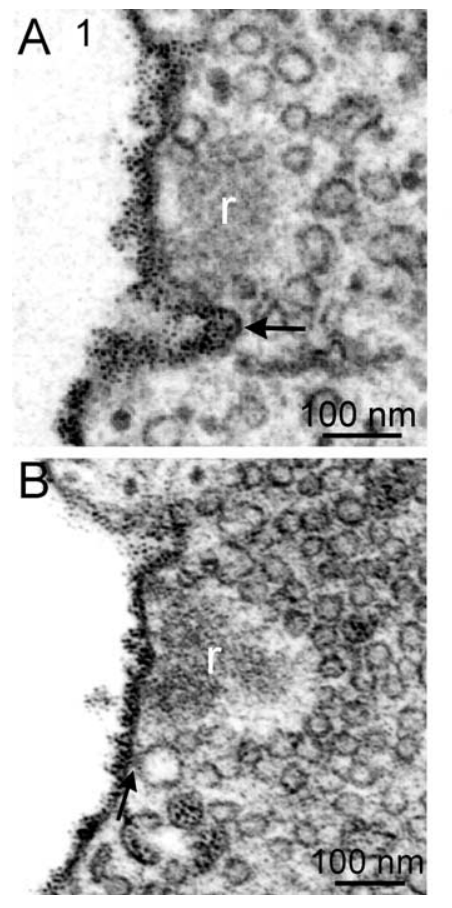

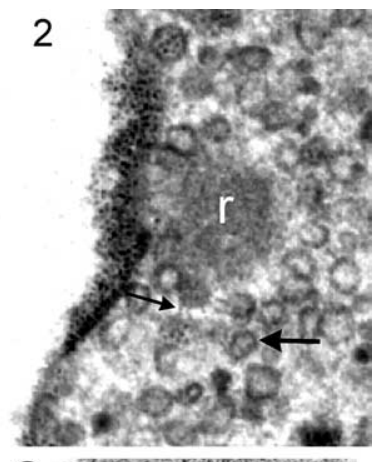

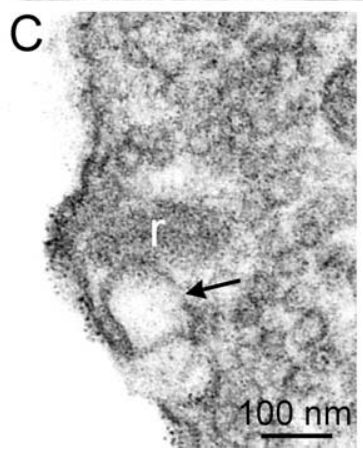

Figure 4. Membrane structures associated with the base of the ribbon in stimulated synaptic terminals. $\boldsymbol{A}$, Two sequential sections through a synaptic ribbon in terminal stimulated in the presence of external cationized ferritin, which decorates the plasma membrane and part of a large invagination (thick arrows). The membrane of the invagination closely associates with the ribbon $(r)$ at contact points indicated by thin arrows. Filamentous tethers similar to those that attach vesicles connect the membrane structure to the ribbon (section 2, thin arrow). $\boldsymbol{B}, \mathrm{A}$ synaptic ribbon in another terminal stimulated in the presence of cationized ferritin, with an unlabeled membrane-bound structure (arrow) connected to the extracellular space via a thin neck. $\boldsymbol{C}$, A synaptic ribbon in a stimulated terminal, with an irregular membrane structure (arrow) associated with the side of the ribbon. Other ferritin-labeled structures visible within the cytoplasm in $\boldsymbol{B}$ and $\boldsymbol{C}$ may represent internalized membrane resulting from previous bouts of exocytosis, because the cells were fixed within $5 \mathrm{~s}$ after the last of a series of several spontaneous calcium spikes.

most of these presumed endocytic structures in the previous studies were not associated with ribbons. Here, we focus on cisternae that are immediately adjacent to ribbons and in some cases directly attached to the surface of the ribbon via filaments.

To learn more about the origin of the large membrane structures associated with the base of ribbons, we fixed bipolar cell synaptic terminals during a series of voltage-clamp depolarizations, while monitoring membrane capacitance as an index of exocytosis and endocytosis. In goldfish bipolar cell terminals, endocytosis after strong stimulation is delayed after stimulation ceases, as revealed by both capacitance measurements and uptake of FM dye (von Gersdorff and Matthews, 1994; Heidelberger, 2001; Rouze and Schwartz, 1998). Also, measurements of glutamate release demonstrate that exocytosis in goldfish bipolar cell terminals ceases rapidly after termination of depolarization (von Gersdorff et al., 1998), which confirms that the plateau of capacitance after strong stimulation reflects absence of endocytosis and not balance of ongoing release and retrieval (Matthews, 1998). Therefore, by monitoring membrane capacitance under voltage clamp and fixing synaptic terminals under conditions unfavorable for endocytosis, we could obtain evidence about whether the large vesicles on the ribbon arise from exocytosis or endocytosis.

Figure 5 shows examples of synaptic ribbons under these conditions in a bipolar terminal fixed by local superfusion of fixative during a series of $250 \mathrm{~ms}$ voltage-clamp pulses from -60 to $0 \mathrm{mV}$,
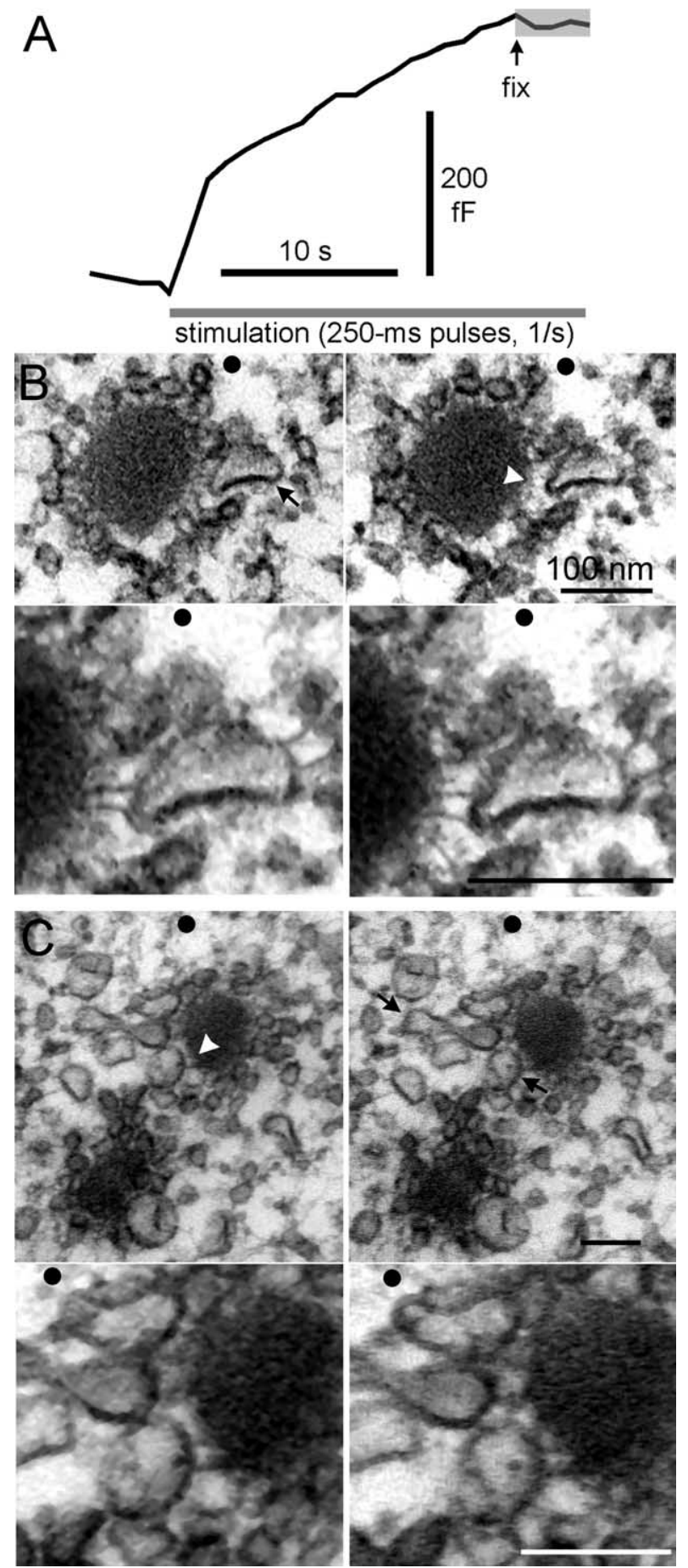

Figure 5. Synaptic ribbons in a bipolar neuron synaptic terminal fixed during repetitive stimulation. $\boldsymbol{A}$, Membrane capacitance increased during a series of $250 \mathrm{~ms}$ depolarizing voltage-clamp pulses. Fixative was applied at the arrow. Baseline capacitance before stimulation was $\sim 2.7 \mathrm{pF}$. Because of fixation-induced increase in membrane conductance, the capacitance values (gray box) after application of the fixative are unreliable. $\boldsymbol{B}, \boldsymbol{C}$, Ribbons in sections parallel to the "footprint" of the terminal on the substrate (section thickness $\sim 90 \mathrm{~nm}$ ). Images are stereo pairs obtained by manually tilting the section. Black arrows indicate examples of large, pleomorphic membranous structures that are contacted by electron-dense filaments (white arrowheads) connecting to the surface of the ribbon. The bottom pair of images in each panel is a higher magnification view of the region containing the bridging filaments. In $\boldsymbol{B}$, the contrast of the filaments in the higher-magnification image was digitally enhanced using Adobe Photoshop 7.0 . Scale bars, $100 \mathrm{~nm}$. The dots in each stereo pair are registration marks to assist in obtaining the stereoscopic depth effect without stereo goggles (to do so, view the figure from a distance of $\sim 40 \mathrm{~cm}$ and alter the convergence of your eyes so that the left and right dots superimpose). 
delivered at $1 / \mathrm{s}$. The evoked change in membrane capacitance neared a plateau by the time of fixation (Fig. $5 A$, arrow). We then examined electron micrographs of thin sections taken parallel to the Aclar substrate, at the attachment footprint of the terminal. We chose this orientation because the flattened surface of the terminal at the footprint allows observation of several ribbons in the same plane and, most importantly, because the filaments that attach vesicles to ribbons are more clearly visible in this orientation than in the more conventional plane of section perpendicular to the plasma membrane.

In the first section contained entirely within the terminal (that is, not including part of the substrate), numerous large, pleomorphic membranous structures were associated with synaptic ribbons (Fig. $5 B, C$, black arrows). In some cases, these were attached to the ribbon by filamentous tethers similar to those that connect normal vesicles to ribbons (Figs. 5B, $C$, white arrowheads). In stereoscopic views, provided by tilting the section, the large structures appeared to extend through the section depth. Therefore, we followed them in the next section into the substrate, which included the plasma membrane, to determine if they extended to the membrane itself. Figure 6 shows the membrane attachment sites of the ribbons of Figure 5, $B$ and $C$, with arrows indicating the same pleomorphic structures that were observed to be tethered
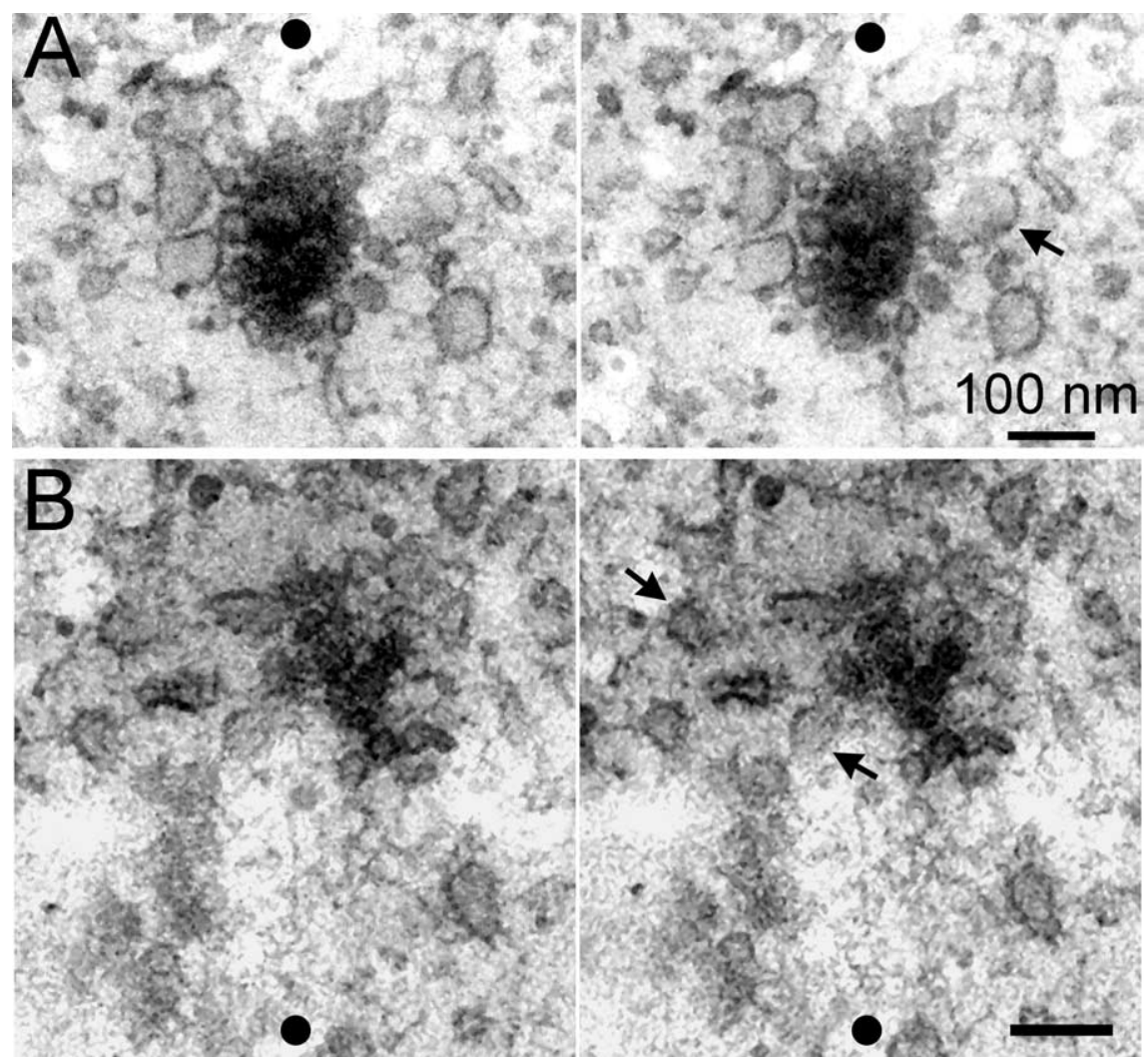

Figure 6. Cisternae connected to synaptic ribbons may connect with the plasma membrane. $A, B$, Images are stereo pairs obtained by manually tilting a section parallel to the plasma membrane, taken at the plane of attachment of the membrane with the Aclar substrate. Because the $90 \mathrm{~nm}$ section at this position includes the substrate, plasma membrane, and a thin layer of cytoplasm, the native contrast was low, and the images are therefore grainy after contrast enhancement using the autocontrast function of Adobe Photoshop 7.0. $\boldsymbol{A}$ and $\boldsymbol{B}$ show the same ribbons illustrated in Figure 5, $B$ and $C$, respectively. Black arrows indicate the continuation of the same membrane structures marked by arrows in Figure 5 . Dots are registration marks for stereoscopic viewing. Scale bars, $100 \mathrm{~nm}$.

to the ribbon in the deeper section. The sec-

tion thickness was $\sim 90 \mathrm{~nm}$, so the large structures extend up the surface of the ribbon $>100 \mathrm{~nm}$ from the plasma membrane.

In the plane that included the plasma membrane, the membrane delimiting the large structures was not visible around the entire perimeter, giving them a horseshoe-like appearance. This is consistent with merging of the two membranes, suggesting that the pleomorphic structures we found to be attached to the ribbon in the deeper section are also continuous with the plasma membrane and therefore open to the extracellular space. To examine this possibility more closely, we turned to transverse sections, perpendicular to the plasma membrane, taken deeper into the terminal in the same stimulated cells. This plane of section complements the information from the plane parallel to the membrane by providing a direct view of membrane invaginations. Consecutive sections through a repetitively stimulated ribbon are shown in Figure $7 A-D$. Large invaginations occupied the region nearest the plasma membrane next to the ribbon, consistent with the pattern shown previously in Figures 5 and 6, and the continuity of the invaginations with the extracellular space is readily apparent (Fig. 7A, arrows).

We quantified the prevalence of the large pleomorphic structures by outlining and measuring the area of each ribbonassociated object, both normal vesicles and larger structures, in sections at the attachment footprint of two terminals fixed during ongoing stimulation and one control terminal dialyzed with ATP- $\gamma \mathrm{S}$. Figure $7 E$ shows the results. At the base of the ribbon (near the plasma membrane), objects with areas $>2000 \mathrm{~nm}^{2}$ (range 2120-12,500 $\mathrm{nm}^{2}$ ) accounted for $17 \%$ of ribbonassociated membranous structures in the stimulated terminals, with the remainder being conventional synaptic vesicles. At the top of the ribbon (sections deeper into the terminal), we found only conventional synaptic vesicles. Few invaginations or pleomorphic structures were observed near ribbons in the presence of ATP- $\gamma$ S. Thus, invaginating structures (1) are markedly increased during repetitive stimulation, (2) are attached to the ribbon by tethers resembling those of synaptic vesicles, and (3) occupy the base of the ribbon nearest the plasma membrane, with which at least some of them are continuous.

Because these cistern-like structures connect to the ribbon at positions that are occupied in resting terminals by synaptic vesicles, we consider it likely that they arose from fusion of these vesicles during repetitive stimulation. In this view, therefore, they are products of exocytosis. Similar pleomorphic cisternae in the vicinity of the ribbon but not attached to it (Fig. $5 C$ ) may be the products of earlier rounds of multivesicular fusion that had detached from the ribbon but had not yet undergone endocytic recycling. We suggest that these large, irregular cisternae are in fact synaptic vesicles that underwent compound exocytosis while attached to the ribbon.

\section{Discussion}

Compound exocytosis: the smoking gun?

A hallmark of the synaptic ribbon is its ability to fuse synaptic vesicles rapidly (Heidelberger et al., 2005; Prescott and Zenisek, 

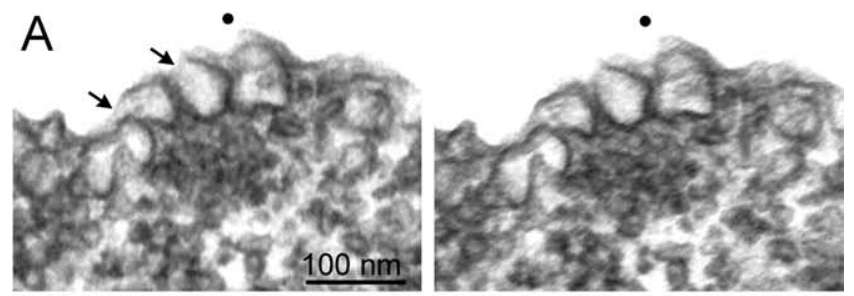

$\mathrm{B}$

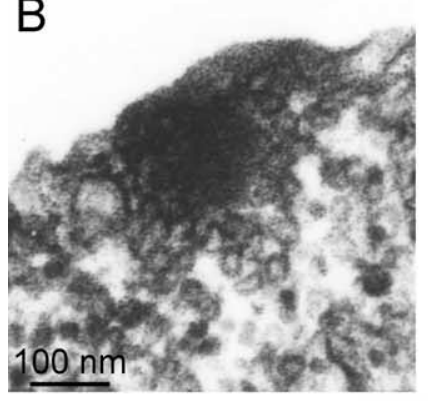

C
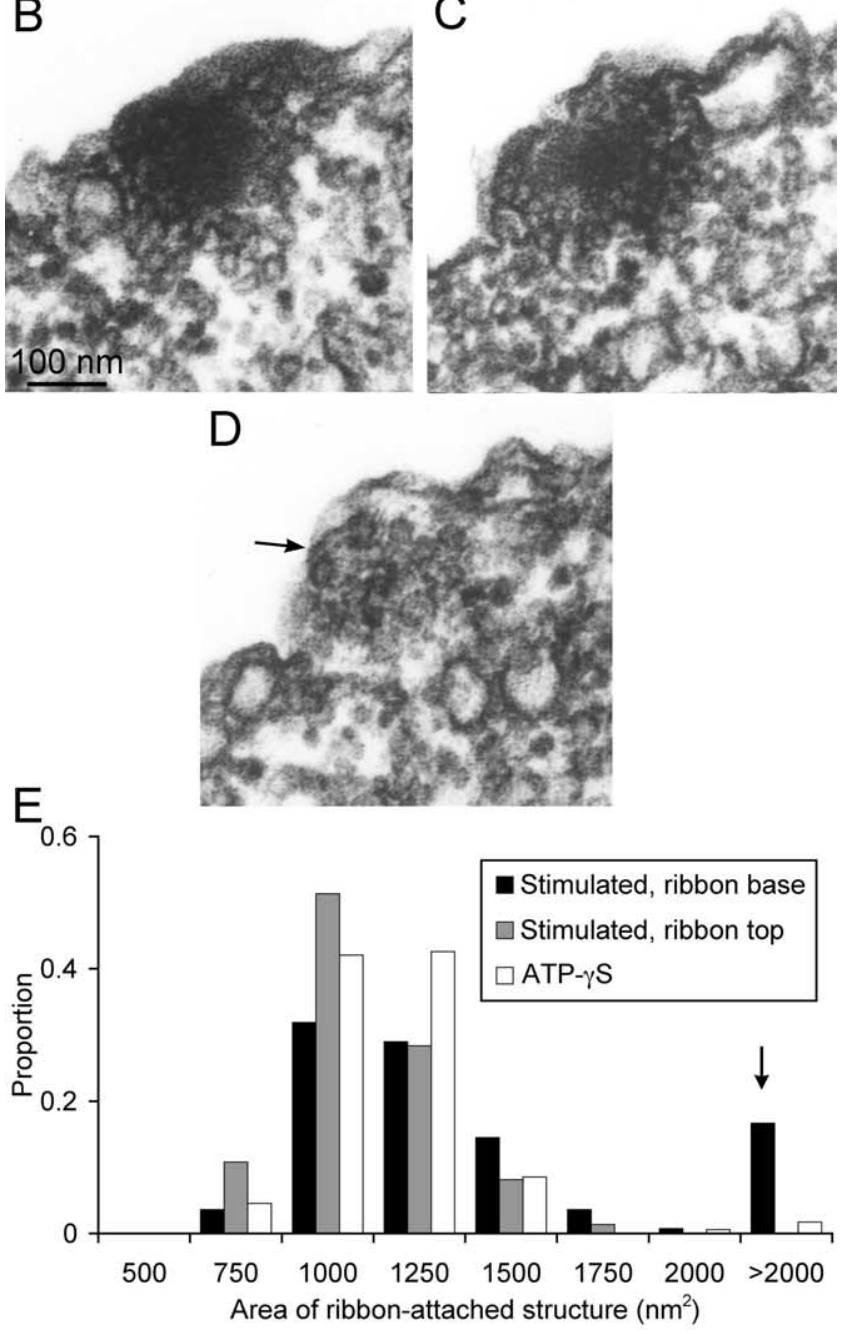

Figure 7. Serial sections through a synaptic ribbon in a bipolar neuron synaptic terminal fixed during repetitive stimulation show that pleomorphic membrane structures are continuous with the extracellular space. $A$, A section in a plane perpendicular to the plasma membrane, at the edge of the ribbon, which is seen in subsequent sections in $\boldsymbol{B}-\boldsymbol{D}$. Images in $\boldsymbol{A}$ are a stereo pair obtained by manually tilting the section. In addition to a cluster of synaptic vesicles at the cytoplasmic end of the ribbon, pleomorphic structures like those shown in Figures 5 and 6 were located at the base of the ribbon, nearest the plasma membrane. In this plane, at least two of the large ribbon-associated structures connect with the extracellular space (arrows). B, C, Images from the next two serial sections, showing the ribbon itself with associated synaptic vesicles. $\boldsymbol{D}$, The next section, at the other edge of the ribbon, shows a cluster of vesicles distal from the plasma membrane and a pleomorphic structure (thin arrow) near the plasma membrane. $\boldsymbol{E}$, Histograms of the size of ribbon-associated membranous structures in sections parallel to the Aclar substrate at the attachment point of the synaptic terminal. Stimulated terminals were fixed during stimulation with $250 \mathrm{~ms}$ voltage-clamp pulses at $1 / \mathrm{s}$, as in Figure $5 \mathrm{~A}$. "Ribbon base" refers to the section nearest the plasma membrane, and "ribbon top" refers to data from a section deeper into the terminal, at the cytoplasmic end of the ribbon. The control terminal was dialyzed with ATP- $\gamma S$ to block neurotransmitter release. Because of the irregular shape of the pleomorphic structures, size was measured by outlining the perimeter of each object and measuring the enclosed area using ImageJ.
2005; Thoreson, 2007). For example, a ribbon in a dissociated bipolar neuron, when strongly depolarized, can fuse $\sim 500$ vesicles s $^{-1}$ (von Gersdorff et al., 1996), and similar rates were found in auditory hair cells (Parsons et al. 1994). Even more startling, when calcium is instantaneously raised by flash photolysis, release can reach $>10^{6}$ vesicles $s^{-1}$ in the terminal as a whole (Heidelberger et al., 1994). The latter result has been deeply puzzling because it is far too fast for vesicles to reach the cell membrane by diffusion. This puzzle would be solved if release of caged calcium rapidly taps vesicles docked at the plasma membrane both at the ribbon and at distant sites where release does not normally occur. However, cross-depletion experiments show that depleting the vesicle pool released by depolarization, likely those at ribbons where calcium channels are located, also abolishes the response to flash photolysis of caged calcium (Heidelberger, 1998). So the puzzle remained as to how ribbons are able to release their vesicles so rapidly.

Presynaptic capacitance measurements in hair cells show that the elementary fusion events are considerably larger than expected for single vesicles (Neef et al., 2007), which suggests that the synaptic ribbon is able to coordinate nearly simultaneous fusion of multiple vesicles. Ribbons in intact circuits also release multiple vesicles simultaneously from hair cells (Glowatzki and Fuchs, 2002) and rod bipolar neurons (Singer et al., 2004). These latter observations of multivesicular release derive from postsynaptic recordings, which also show that several vesicles released together do not saturate the postsynaptic receptors. Thus, multivesicular release seems to occur at ribbon synapses and can be functionally significant.

One mechanism postulated to explain the puzzling characteristics of release at ribbons, including multivesicular release, is "compound fusion," in which vesicles fuse with other vesicles either sequentially in a wave that advances rapidly up the ribbon (Parsons and Sterling, 2003), or by coalescence of two or more vesicles before fusion with the plasma membrane (Singer et al., 2004). This mechanism could empty multiple vesicles without requiring them to move to the presynaptic membrane. Compound fusion has been observed optically in non-neural cells (Alvarez de Toledo and Fernandez, 1990; Leung et al., 2002; Chen et al., 2005; Kishimoto et al., 2006; Pickett and Edwardson, 2006); however, the single fact of temporally coincident fusion observed electrophysiologically at the ribbon synapse does not establish the mechanism. In short, at the ribbon, there was a "motive" (speed) and a "body" (multivesicular release), but no "smoking gun" (high-resolution images of multiply fused vesicles on the ribbon promptly following a bout of exocytosis). We suggest that the large membrane structures tethered to ribbons in our experiments represent that smoking gun.

If compound fusion were the mechanism underlying multivesicular release, there should be (1) fewer vesicles attached to the ribbon, (2) more large vesicles because of their coalescence, and (3) continuity between the lumen of vesicles attached to the ribbon and the extracellular space. Indeed, prolonged bouts of vesicle release reduced vesicle density on ribbons by $\sim 50 \%$, compared with cells with exocytosis blocked by dialysis with ATP- $\gamma \mathrm{S}$ (Fig. 2E). This represents a lower bound for the actual degree of vesicle depletion from ribbons because the time between stimulation and effective fixation might have allowed some recovery. Holt et al. (2004) also reported that bipolar cells fixed within seconds after prolonged depolarization had fewer vesicles docked at the presynaptic membrane, but no change in the total number of vesicles on the ribbon. This difference from our result might arise from different stimulus conditions. We used spontaneous 
calcium action potentials ( $<200 \mathrm{~ms}$ in duration) (Zenisek and Matthews, 1998), $250 \mathrm{~ms}$ depolarizations under voltage clamp, or brief puffs of high external potassium, whereas Holt et al. (2004) used $1 \mathrm{~min}$ of high external potassium. The latter would prolong the elevation of $\mathrm{Ca}^{2+}$ and possibly reduce depletion either by accelerating reloading or because the release rate adapts.

\section{Large cisternae attached to ribbons: a product of endocytosis or exocytosis?}

Repeated bouts of exocytosis not only depleted the ribbons of tethered vesicles, they also increased the proportion of larger vesicles (Fig. 3). Furthermore, membranous cisternae could be traced from the plasma membrane to sites of attachment on the ribbon's face (Figs. 5, 6). These attachments were to the ribbon's basal region near the plasma membrane, where exocytosis is expected, and were mediated by filaments similar to those that attach synaptic vesicles to the ribbon. This static image could logically represent endocytosis: membrane being retrieved and repackaged into larger vesicles at the base, which then pinch off smaller vesicles that populate the distal ribbon. This alternative would suggest that the ribbon is at least partly an endocytosis machine, because crucial locations at the base of the ribbon would be occupied with endocytic processing to the exclusion of further exocytosis. However, during vigorous stimulation, endocytosis is thought to be suppressed in goldfish bipolar cells (von Gersdorff and Matthews, 1994; Rouze and Schwartz, 1998; Heidelberger, 2001). Because the cells in our experiments were fixed under this condition and because the cisternae occupy locations where neurotransmitter release should occur, we suggest that the ribbon-associated cisternae likely represent exocytosis.

If they are exocytic rather than endocytic, the large cistern-like objects associated with ribbons could correspond to the large endosomes reported previously by Coggins et al. (2007) to undergo exocytosis during stimulation in goldfish bipolar cell terminals. That is, the ribbon-attached cisternae could be endosomes created by bulk endocytosis (Paillart et al., 2003; Holt et al., 2004), which migrated to ribbons and somehow displaced normal synaptic vesicles in the part of the ribbon nearest the plasma membrane.

An attractive alternative, however, is that the cisternae are created during exocytosis at the base of the ribbon. Under this scenario, the large vesicles on the ribbon shown in Figure 3, which are more prevalent after calcium influx, arise from fusion of two or more adjacent vesicles attached to the ribbon, without fusing to the plasma membrane. This has been observed in nonneuronal cells (Pickett and Edwardson, 2006). Such vesiclevesicle fusion might be triggered during depolarization by the local elevation of $\mathrm{Ca}^{2+}$ via channels in the plasma membrane at the base of the ribbon. Similarly, the large cistern-like structures attached to the ribbon, yet continuous with the plasma membrane, may represent vesicles that fused sequentially with other vesicles that had previously undergone exocytosis. In electron micrographs, vesicles attached to ribbons are tightly packed and appear to touch (Figs. $2 A-C, 3 A$ ). In addition to vesicle-SNAREs (soluble $N$-ethylmaleimide-sensitive factor attachment protein receptors), vesicles also contain target-SNARE isoforms (WalchSolimena et al., 1995; Takamori et al., 2006), which could mediate formation of fusion complexes between vesicles. Homotypic fusion of this sort is known to occur among secretory granules in other cellular contexts (Alvarez de Toledo and Fernandez, 1990; Leung et al., 2002; Chen et al., 2005; Kishimoto et al., 2006). Also, vesicles at ribbon synapses may be specialized for such compound fusion, perhaps by incorporation of novel isoforms of
SNARE proteins not found in vesicles of conventional synapses. Seen in this light, the ribbon itself may be thought of as a cytoskeletal component that stabilizes fused vesicles so that they are available for subsequent fusion with other vesicles deeper into the cell. Testing this possibility in living cells may be feasible using fluorescent labeling of vesicles (Zenisek et al., 2000) and ribbons (Zenisek et al., 2004; LoGiudice et al., 2008; Zenisek, 2008).

In any case, the long-standing question as to how vesicles on the synaptic ribbon rapidly empty their contents may now have an answer: by compound exocytosis.

\section{References}

Alvarez de Toledo G, Fernandez JM (1990) Compound versus multigranular exocytosis in peritoneal mast cells. J Gen Physiol 95:397-409.

Chen Y, Warner JD, Yule DI, Giovannucci DR (2005) Spatiotemporal analysis of exocytosis in mouse parotid acinar cells. Am J Physiol Cell Physiol 89:C1209-C1219.

Coggins MR, Grabner CP, Almers W, Zenisek D (2007) Stimulated exocytosis of endosomes in goldfish retinal bipolar neurons. J Physiol (Lond) 584:853-865.

Glowatzki E, Fuchs PA (2002) Transmitter release at the hair cell ribbon synapse. Nat Neurosci 5:147-154.

Heidelberger R (1998) Adenosine triphosphate and the late steps in calcium-dependent exocytosis at a ribbon synapse. J Gen Physiol 111:225-241.

Heidelberger R (2001) ATP is required at an early step in compensatory endocytosis in synaptic terminals. J Neurosci 21:6467-6474.

Heidelberger R, Matthews G (1992) Calcium influx and calcium current in single synaptic terminals of goldfish retinal bipolar neurons. J Physiol (Lond) 447:235-256

Heidelberger R, Heinemann C, Neher E, Matthews G (1994) Calcium dependence of the rate of exocytosis in a synaptic terminal. Nature 371:513-515.

Heidelberger R, Sterling P, Matthews G (2002) Roles of ATP in depletion and replenishment of the releasable pool of synaptic vesicles. J Neurophysiol 88:98-106.

Heidelberger R, Thoreson WB, Witkovsky P (2005) Synaptic transmission at retinal ribbon synapses. Prog Retin Eye Res 24:682-720.

Holt M, Cooke A, Neef A, Lagnado L (2004) High mobility of vesicles supports continuous exocytosis at a ribbon synapse. Curr Biol 14:173-183.

Kishimoto T, Kimura R, Liu TT, Nemoto T, Takahashi N, Kasai H (2006) Vacuolar sequential exocytosis of large dense-core vesicles in adrenal medulla. EMBO J 25:673-682.

Lenzi D, Crum J, Ellisman MH, Roberts WM (2002) Depolarization redistributes synaptic membrane and creates a gradient of vesicles on the synaptic body at a ribbon synapse. Neuron 36:649-659.

Leung YM, Sheu L, Kwan E, Wang G, Tsushima R, Gaisano H (2002) Visualization of sequential exocytosis in rat pancreatic islet beta cells. Biochem Biophys Res Commun 292:980-986.

LoGiudice L, Sterling P, Matthews G (2008) Mobility and turnover of vesicles at the synaptic ribbon. J Neurosci 28:3150-3158.

Matthews G (1998) A lie detector test for presynaptic capacitance measurements. Neuron 21:940-941.

Neef A, Khimich D, Pirih P, Riedel D, Wolf F, Moser T (2007) Probing the mechanism of exocytosis at the hair cell ribbon synapse. J Neurosci 27:12933-12944.

Paillart C, Li J, Matthews G, Sterling P (2003) Endocytosis and vesicle recycling at a ribbon synapse. J Neurosci 23:4092-4099.

Parsons TD, Sterling P (2003) Synaptic ribbon conveyor belt or safety belt? Neuron 37:379-382.

Parsons TD, Lenzi D, Almers W, Roberts WM (1994) Calcium-triggered exocytosis and endocytosis in an isolated presynaptic cell: capacitance measurements in saccular hair cells. Neuron 13:875-883.

Pickett JA, Edwardson JM (2006) Compound exocytosis: mechanisms and functional significance. Traffic 7:109-116.

Prescott ED, Zenisek D (2005) Recent progress towards understanding the synaptic ribbon. Curr Opin Neurobiol 15:431-436.

Rao-Mirotznik R, Harkins AB, Buchsbaum G, Sterling P (1995) Mammalian rod terminal: architecture of a binary synapse. Neuron 14:561-569.

Rouze NC, Schwartz EA (1998) Continuous and transient vesicle cycling at a ribbon synapse. J Neurosci 18:8614-8624. 
Schaeffer SF, Raviola E (1978) Membrane recycling in the cone cell endings of the turtle retina. J Cell Biol 79:802-825.

Siegel S (1956) Nonparametric statistics for the behavioral sciences. New York: McGraw-Hill.

Singer JH, Lassova L, Vardi N, Diamond JS (2004) Coordinated multivesicular release at a mammalian ribbon synapse. Nat Neurosci 7:826-833.

Smith JE, Reese TS (1980) Use of aldehyde fixatives to determine the rate of synaptic transmitter release. J Exp Biol 89:19-29.

Sterling P, Matthews G (2005) Structure and function of ribbon synapses. Trends Neurosci 28:20-29.

Takamori S, Holt M, Stenius K, Lemke EA, Gronborg M, Riedel D, Urlaub H, Schenck S, Brugger B, Ringler P, Muller SA, Rammner B, Grater F, Hub JS, De Groot BL, Mieskes G, Moriyama Y, Klingauf J, Grubmuller H, Heuser J, et al. (2006) Molecular anatomy of a trafficking organelle. Cell 127:831-846.

Thoreson WB (2007) Kinetics of synaptic transmission at ribbon synapses of rods and cones. Mol Neurobiol 36:205-223.

von Gersdorff H, Matthews G (1994) Inhibition of endocytosis by elevated internal calcium in a synaptic terminal. Nature 370:652-655. von Gersdorff H, Vardi E, Matthews G, Sterling P (1996) Evidence that vesicles on the synaptic ribbon of retinal bipolar neurons can be rapidly released. Neuron 16:1221-1227.

von Gersdorff H, Sakaba T, Berglund K, Tachibana M (1998) Submillisecond kinetics of glutamate release from a sensory synapse. Neuron 21:1177-1188.

Walch-Solimena C, Blasi J, Edelmann L, Chapman ER, von Mollard GF, Jahn $\mathrm{R}$ (1995) The t-SNAREs syntaxin 1 and SNAP-25 are present on organelles that participate in synaptic vesicle recycling. J Cell Biol 128:637645.

Zenisek D (2008) Vesicle association and exocytosis at ribbon and extraribbon sites in retinal bipolar cell presynaptic terminals. Proc Natl Acad Sci USA 105:4922-4927.

Zenisek D, Matthews G (1998) Calcium action potentials in retinal bipolar neurons. Vis Neurosci 15:69-75.

Zenisek D, Steyer JA, Almers W (2000) Transport, capture and exocytosis of single synaptic vesicles at active zones. Nature 406:849-854.

Zenisek D, Horst NK, Merrifield C, Sterling P, Matthews G (2004) Visualizing synaptic ribbons in the living cell. J Neurosci 24:9752-9759. 